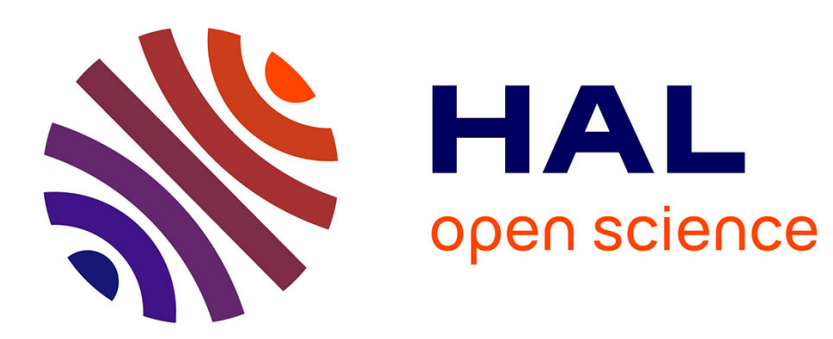

\title{
Effect of deformation on knitted glass preform in-plane permeability
}

Gilles Dusserre, Eric Jourdain, Gérard Bernhart

\section{To cite this version:}

Gilles Dusserre, Eric Jourdain, Gérard Bernhart. Effect of deformation on knitted glass preform in-plane permeability. Polymer Composites, 2011, 32 (1), p.18-28. 10.1002/pc.21011 . hal-01851416

\section{HAL Id: hal-01851416 https://hal.science/hal-01851416}

Submitted on 6 Nov 2018

HAL is a multi-disciplinary open access archive for the deposit and dissemination of scientific research documents, whether they are published or not. The documents may come from teaching and research institutions in France or abroad, or from public or private research centers.
L'archive ouverte pluridisciplinaire HAL, est destinée au dépôt et à la diffusion de documents scientifiques de niveau recherche, publiés ou non, émanant des établissements d'enseignement et de recherche français ou étrangers, des laboratoires publics ou privés. 


\title{
Effect of Deformation on Knitted Glass Preform In-Plane Permeability
}

\author{
Gilles Dusserre, ${ }^{1,2}$ Eric Jourdain, ${ }^{1,2}$ Gérard Bernhart ${ }^{1,2}$ \\ ${ }^{1}$ Université de Toulouse; INSA, UPS, Mines Albi, ISAE; ICA (Institut Clément Ader); Campus Jarlard, \\ F-81013 Albi cedex 09, France
}

${ }^{2}$ Ecole des Mines Albi, Centre CROMeP, Campus Jarlard, F-81013 Albi, France

\begin{abstract}
The excellent processing properties of knitted preforms for composite applications, as formability and permeability, are not sufficient to compensate the poor mechanical characteristics of the resulting material. Initial preform deformation is a way to improve these properties, but it modifies the permeability and changes the optimal infiltration conditions. This article presents an experimental setup and discusses the reliability of the permeability measurements. Experimental results show that the course-wise permeability is significantly modified by the deformation of the fabric, whereas the wale-wise permeability is quite insensitive to the deformation. In an equivalent isotropic system, the deformation essentially influences the anisotropy ratio.
\end{abstract}

\section{INTRODUCTION}

Knitted fabrics exhibit excellent properties for the processing of composite materials by injection or infusion. They are net-shape manufactured (minimizing the material wastage) with a high level of automation and complex shapes (hemispherical surfaces, revolution parts without joints) can be provided directly with geometric singularities [1] like holes or thickness variation. Their high deformation ability increases the complexity of the possible shapes [2] and makes the manual draping easier. This crucial stage requires less dexterity, decreasing the dispersions on the final material properties. The knitting process provides the possibility to obtain different loop structures and to integrate various fiber types in a same component, and thicknesses and mechanical properties can be affected on selected areas. Moreover, most of the fibers used for composite material manufacturing (glass,

Correspondence to: G. Dusserre; e-mail: Gilles.Dusserre@mines-albi.fr carbon, aramid, and basalt) are available in a knittable configuration. Finally, knitted fabrics exhibit often low fiber volume fraction and high permeability compared with woven fabrics, resulting in a faster impregnation process. The use of dissipation media is not required any more and draping stage duration is decreased.

Nevertheless, the use of such reinforcements for composite manufacturing is still limited because of the general poor mechanical properties of the resulting material. The high curvature of the yarns leads to a very weak apparent elasticity modulus, and the very aerated structure of the knitted fabrics does not allow to reach a high fiber volume fraction in the final material. The most interesting mechanical property of these composites is the limited loss of compression strength after impact, but it is not sufficient to compensate the poor properties of the non impacted material. Last but not least, the knitting process, very mechanized, creates interlocked loops, thanks to the needles hooks leading often to the rupture of many filaments during preform knitting, and contributing to decrease of fabric rupture and rigidity properties.

Mechanical properties improvement of knitted fabric reinforced composites is mandatory to extend their use to low-structural complex shaped aeronautical parts. To reach this goal, investigations could follow two strategies: either at fabric level by

- Improving the rigidity of the knitted structure by interlocking low bended fibers,

- Finding the best fiber material with a good knit ability, good mechanical properties and low density and cost [3], or at injection process level by

- Compacting the preform in the mould to increase the fiber volume fraction. Optimizing the deformation of the preform during draping stage to obtain higher initial elasticity modulus.

This last route has been investigated by a few authors, showing that tensile and compressive properties of the composite are affected by initial preform deformation, 
before resin transfer molding. According to Leong et al. [4], the uniaxial stretching of the knitted fabric induces a fiber alignment in the loading direction that improves the tensile strength and stiffness. Khondker et al. [5] have reported a wale-wise composite modulus improvement arising $10 \%$ after a $10 \%$ wale-wise stretching of a Milano weft-knit reinforcement. In the case of biaxial preform stretching [5], the composite compressive properties do not change significantly. Nevertheless, the effect on tensile properties due to the change of the fiber orientation is very difficult to relate to the initial biaxial deformation level.

Optimization of knitted fabric processing conditions by injection or infusion can be achieved using numerical tools involving Darcy flow simulation. This requires a proper measurement of preform permeability that is supposed to be a porous media. Because of the difficulty to model the relationship between meso- and macroscale resin flows in such complex structures [6], it is very difficult to foresee the permeability of a knitted preform and its evolution with the deformation. Woven [7, 8] and knitted $[9,10]$ fabric mechanical behavior in wale- and course-wise extensions have been studied and are well understood. Experimental works investigating the relationship between permeability and preform deformation are mainly related to transverse compression [7, 11] and inplane shearing [12-16]. Moreover, recent developments in multiscale flow modeling in the unit cell [17-21] seem to be promising tools for permeability prediction of deformed reinforcements [22-24], and may be also useful for knitted mesostructures.

This article mainly addresses the experimental relationship between in-plane tensile deformation and knitted reinforcement permeability with the aim to control and then optimize impregnation processes, and further to contribute to mechanical property reproducibility and/or optimization. Setup complexity and textile heterogeneity induce a significant dispersion of permeability values. Thus, a large part of the paper focuses on measurements reliability to ensure the validity of the results.

\section{MATERIAL AND EXPERIMENTAL SETUP}

\section{Permeability Measurements}

Permeability $K$ relates the average fluid velocity $\langle\boldsymbol{V}\rangle$ of a fluid flowing through a porous medium to its viscosity $\mu$ and to the fluid pressure gradient $\nabla \boldsymbol{P}$. This relation, known as the Darcy's law (Eq. 1) [25], has been demonstrated experimentally and is valid in steady state flow conditions, assuming fluid incompressibility, perfectly rigid porous medium and no inertia effects (Reynolds number lower than 1).

$$
\langle\boldsymbol{V}\rangle=\frac{K}{\mu} \nabla \boldsymbol{P} .
$$

This equation has been formulated by plotting the hydrostatic pressure against the flow rate, leading to a steady state value of the permeability of the saturated porous medium. This value is an intrinsic characteristic of the porous medium and is widely used in many applications. Nevertheless, if this method is quite useful to measure through-thickness permeability of fibrous media (particular case of thin porous media), the result is not representative of the infiltration phenomena involved in the processing of textile composites. Textile reinforcements are dual scale porous media, involving two different velocity flowing processes, inside and around the tows [26-28] due to capillary effects. This implies a difference between permeability values measured for saturated and unsaturated media. That is the reason why many authors prefer a flow front monitoring method providing permeability values reliable for mold filling time prediction. By integrating the Darcy's law under constant pressure loss in the fibrous media, the permeability can be calculated directly from the evolution of the flow front position with time. This method can be applied in 1D [29, 30], 2D [23, $31,32]$, or 3D [33, 34] configurations, increasing the simultaneous measurement capabilities but also experimental and parameter identification complexity [35].

In the present case, a $2 \mathrm{D}$ method was preferred to ensure the measurement of both course- and wale-wise permeability values for a given knitted fabric deformation configuration. A 3D method was not necessary here because of the thinness of the fabric compared with the in-plane dimensions and its large porosity. Through-thickness flow can then be neglected, as well as capillary effects (cf. next section).

\section{Fibrous Fabric Reinforcement}

A $1 \times 1$ rib weft-knitted fabric was investigated. It was knitted from two 300 tex multifilament E-glass yarns (constituted on 1,800 16- $\mu$ m-diameter filaments, leading to a 0.71 -mm-diameter yarn) on a 5.2-gauge Stoll knitting machine leading to a $2,400 \mathrm{~g} \cdot \mathrm{m}^{-2}$ area density fabric. The $200 \times 200 \mathrm{~mm}^{2}$ samples have a thickness of $4.4 \mathrm{~mm}$ under a $5-\mathrm{kPa}$ pressure. Their size is large enough to provide sufficient data for permeability identification, but not to large to ensure deformation homogeneity. The $1 \times 1$ rib knit is an interesting case study to understand the phenomena involved during infiltration: it is the simplest double needle board loop structure, and no wale-axis curling occurs at the contrary of simple needle board structures [36]. The elementary cell (cf. Fig. 1), made by two opposite needles in the same course, has a mean size about $1.6 \mathrm{~mm}$ in the wale-wise direction (length between two consecutives yarn crossover points) and $3.5 \mathrm{~mm}$ in the course-wise direction (width of two wales) and uses a yarn length of $22 \mathrm{~mm}$. The theoretical fiber volume fraction of this knitted fabric evaluated from glass density and sample mass and size is close to $22 \%$. The actual fiber volume fraction value is quite higher because of the compaction of the reinforcement when placed on the experimental setup (27\% for 3.5-mm-thick sample). 


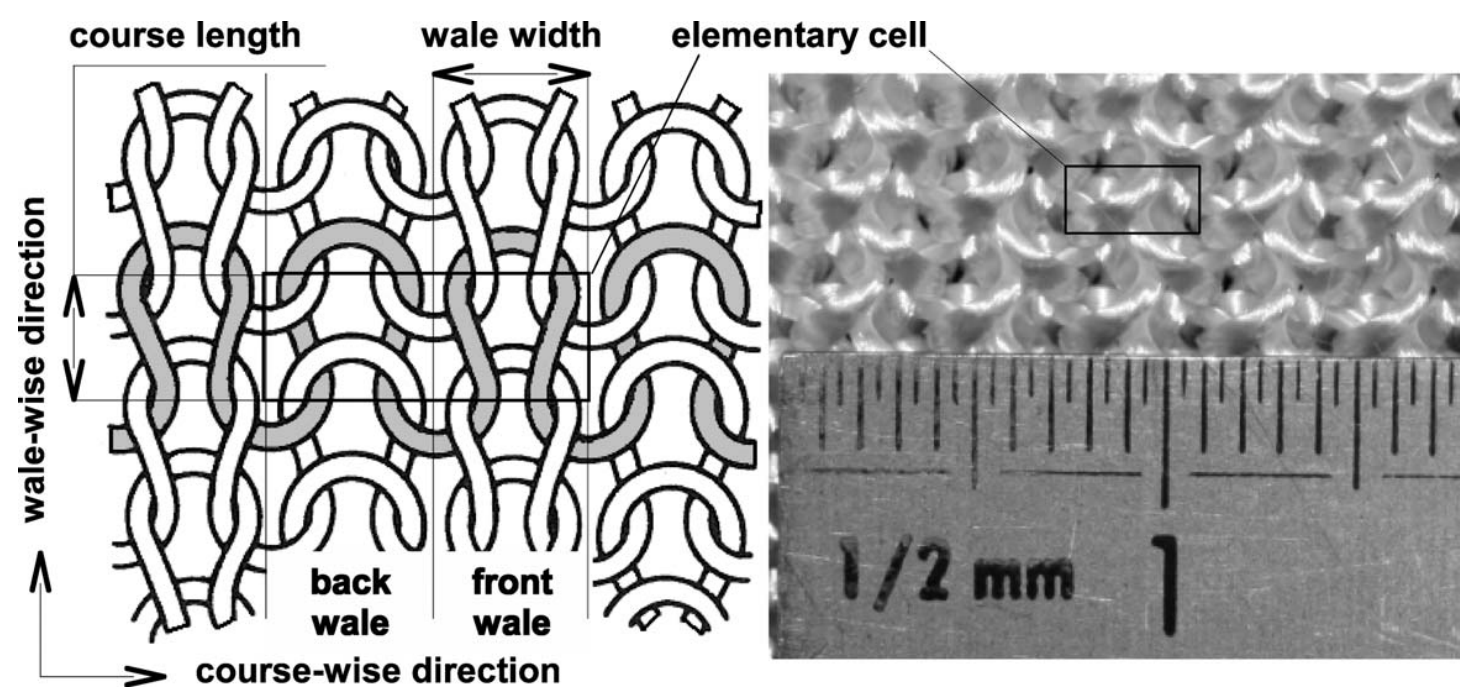

FIG. 1. Schematic diagram of the $1 \times 1$ rib structure.

Mechanical tests have been performed on such structures and are reported elsewhere [3]. Figure 2 reports a typical course-wise and wale-wise load-deformation loop that shows the high permanent deformation levels $(20 \%)$ reached after application of a same low load level (0.5 $\mathrm{N} \cdot \mathrm{mm}^{-1}$ ). Such load levels are easily reached during draping operations and under similar load, the coursewise deformation is approximately twice the wale-wise one. Because of sample and experimental setup limited size, these deformation levels could not be reached during infusion tests. Thus, deformation values of $7.5 \%$ (wale) and $15 \%$ (course) were selected, taking into account the size of sample and permeability setup.

\section{Infiltration Fluid}

The knitted preforms are expected to be impregnated with epoxy resin. One of the most widely used epoxy resin for aeronautical applications is the HexFlow ${ }^{\circledR}$ RTM6. The surface tension of this resin is about 32 $\mathrm{mN} \cdot \mathrm{m}^{-1}$ [37], and its viscosity at processing temperature $\left(100^{\circ} \mathrm{C}\right)$ about $0.06 \mathrm{~Pa} \cdot \mathrm{s}$, but this viscosity is widely dependent on time and temperature due to polymerization phenomena. According to Ma and Shishoo [38], the fluid does not play a significant role on the value of the permeability measurement, especially if viscosities are of the same order of magnitude. Nevertheless, surface tension is also a critical property as it governs capillary effects inside the tows [26-28], and a permeability dependence to fluid nature may be observed, especially for high fiber volume fraction [39].

Nevertheless, many authors use inert fluid such as corn syrup [38, 40] or silicone oil [11]. In this work, canola oil with a room temperature viscosity between 0.05 and $0.075 \mathrm{~Pa} \cdot \mathrm{s}$ and surface tension of $33.4 \pm 0.1 \mathrm{mN} \cdot \mathrm{m}^{-1}$ was selected, close to thermoset resin typical values. The advantages of this fluid are that it does not require any heating, is easy to clean, and creates no occupational health and safety problem. Moreover, the Newtonian behavior of this oil has been checked to be perfect for shear rates up to $10^{3} \mathrm{~s}^{-1}$. The shear rate in the tests, estimated as the ratio between the velocity of the flow front and the thickness of the reinforcement, is between 1 and $50 \mathrm{~s}^{-1}$ corresponding to a Reynolds number between $2 \times$ $10^{-3}$ and $10^{-2}$. As a consequence, inertia can be neglected compared to viscosity contribution, but capillary effects have to be discussed in relation with results. The only drawback of this fluid is its temperature-dependent viscosity (Fig. 3), established experimentally with a 60mm-diameter plate rheometer; but its perfect Arrhenian behavior makes it easy to take into account for isothermal tests.

\section{Experimental Setup}

The selected knitted reinforcements impregnation process is between light resin transfer molding (LRTM) and

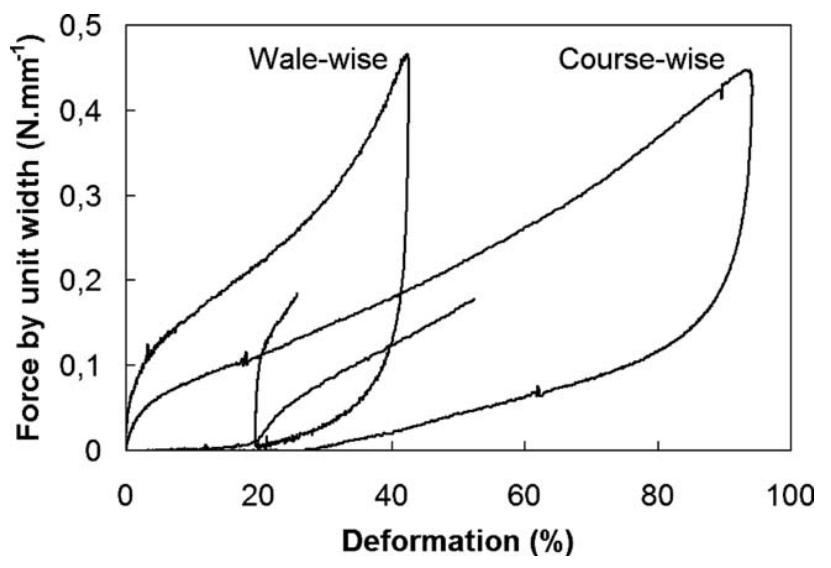

FIG. 2. Cyclic tensile tests performed in wale- and course-wise direction on $1 \times 1$ rib knit samples. 


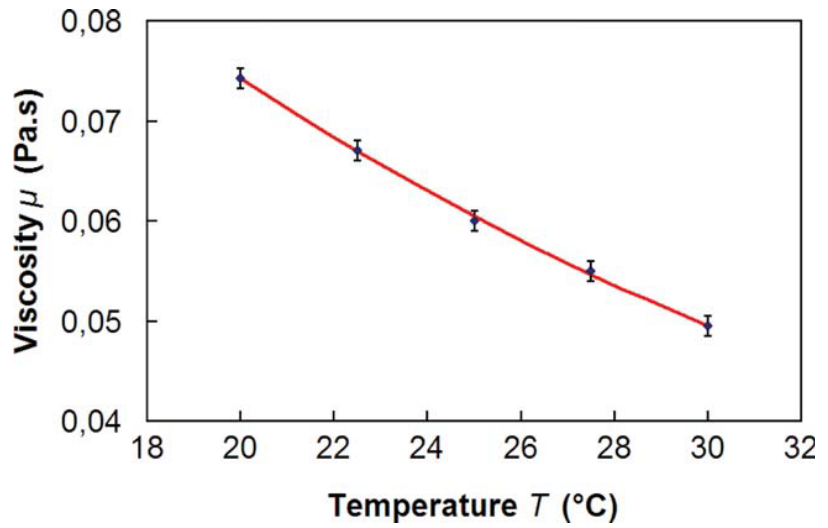

FIG. 3. Temperature dependence of the canola oil viscosity. [Color figure can be viewed in the online issue, which is available at wileyonlinelibrary.com.]

vacuum-assisted resin infusion (VARI) with in-plane resin flow and only vacuum bagging. Permeability measurements are performed using a bidirectional method in the same conditions as involved in the actual infusion process. This bidirectional method is particularly interesting in the present case because both course- and wale-wise measurements are performed in the same experiment. The $200 \times 200 \mathrm{~mm}^{2}$ knitted reinforcement is deposited on a flat metallic plate to allow an accurate thickness measurement using a $0.01-\mathrm{mm}$ resolution comparator. A vacuum pressure lower than $10 \mathrm{~Pa}$ is applied under a $0.08 \pm$ 0.005-mm-thick nylon vacuum bagging using a vacuum pump and a central injection inlet feeds the fluid into the knitted fabric, via a $10-\mathrm{mm}$ diameter pipe. The initial fluid level in the tank is set to the same height as the sample to minimize the hydrostatic pressure. A CCD camera perpendicular to the knitted fabric monitors the flow front position (Fig. 4).

\section{Permeability determination}

Darcy's law analytical formulation in a two-dimensional anisotropic fibrous medium. In the case of a $2 \mathrm{D}$ isotropic radial flow, the mass conservation equation can be integrated in a cylindrical coordinates system to obtain the expression of the pressure gradient as a function of the boundary conditions (atmospheric pressure at injection inlet radius $R_{0}$ and vacuum pressure at flow front radius $R_{\mathrm{f}}$ ). By integrating the Darcy's law (Eq. l), Eq. 2 is obtained [41] that relates $R_{0}, R_{\mathrm{f}}$, pressure drop $\delta P$, fluid viscosity $\mu$, fiber volume fraction $V_{\mathrm{f}}$, permeability $K$, and time $t$.

$$
F\left(R_{\mathrm{f}}, R_{0}\right)=R_{\mathrm{f}}^{2}\left[2 \cdot \ln \left(\frac{R_{\mathrm{f}}}{R_{0}}\right)-1\right]+R_{0}^{2}=\frac{4 \delta P}{\mu\left(1-V_{\mathrm{f}}\right)} K t .
$$

In the case of an anisotropic medium, Darcy's law integration is not possible analytically without taking into account an equivalent isotropic system [42] in a coordinate system parallel to the principal directions of permeability tensor, corresponding in the present case to course- and wale-wise directions. This method states that the courseand wale-wise flow front positions, respectively, $R_{\mathrm{fC}}$ and $R_{\mathrm{fW}}$, can be transformed into the same isotropic equivalent flow front position $R_{\mathrm{fe}}$ by introducing two parameters $\xi$ and $\eta(E q .3)$, and that the course- and wale-wise permeability can be expressed as a function of the equivalent isotropic permeability $K_{\mathrm{e}}$ and the same parameters (Eq.4).

$$
\begin{gathered}
\xi R_{\mathrm{fC}}=\eta R_{\mathrm{fW}}=R_{\mathrm{fe}} . \\
\xi^{2} K_{\mathrm{C}}=\eta^{2} K_{\mathrm{W}}=K_{\mathrm{e}} .
\end{gathered}
$$

Among the infinity of possibilities, $\xi$ and $\eta$ are chosen as a function of the anisotropy ratio, $\alpha$, between the waleand the course-wise permeability.

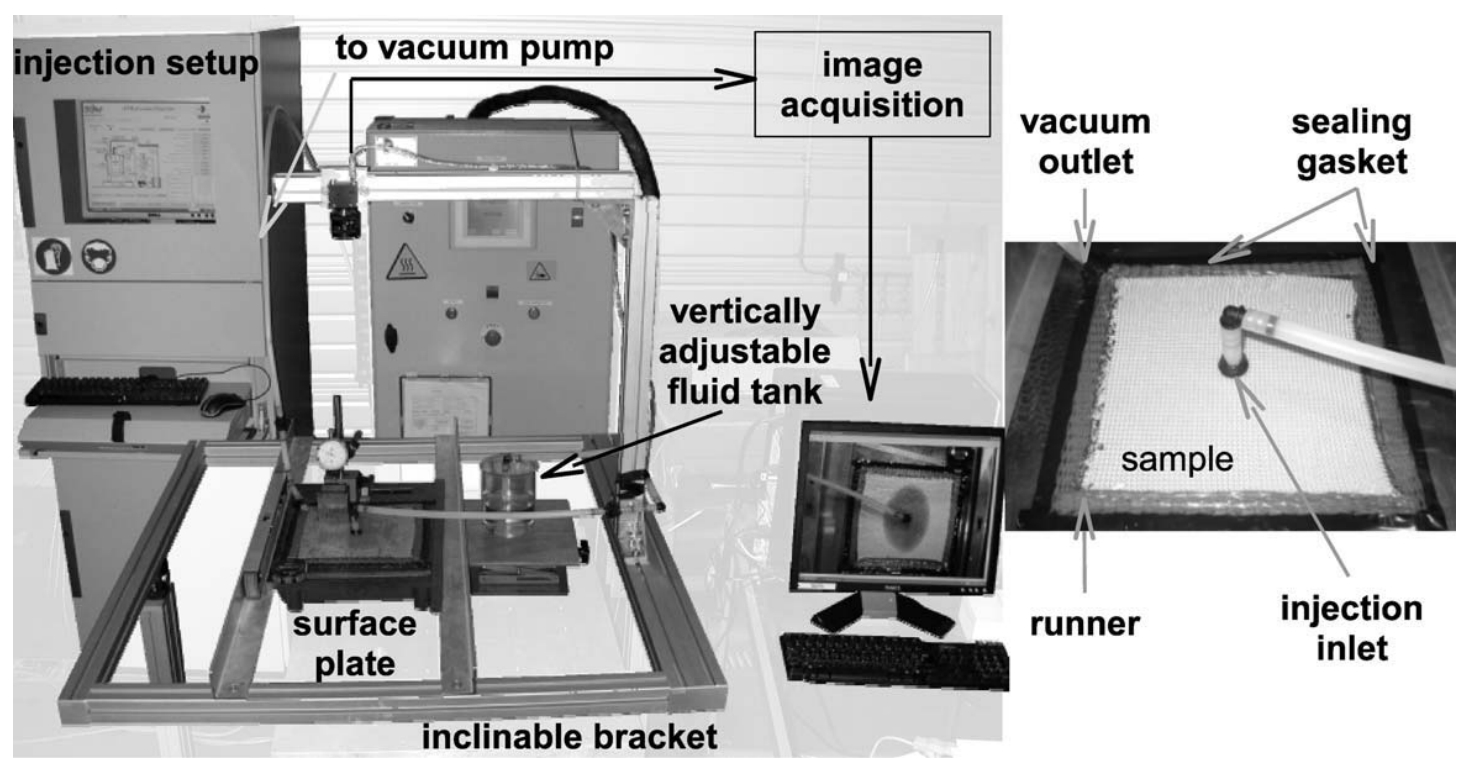

FIG. 4. Experimental setup: general view and sample view. 

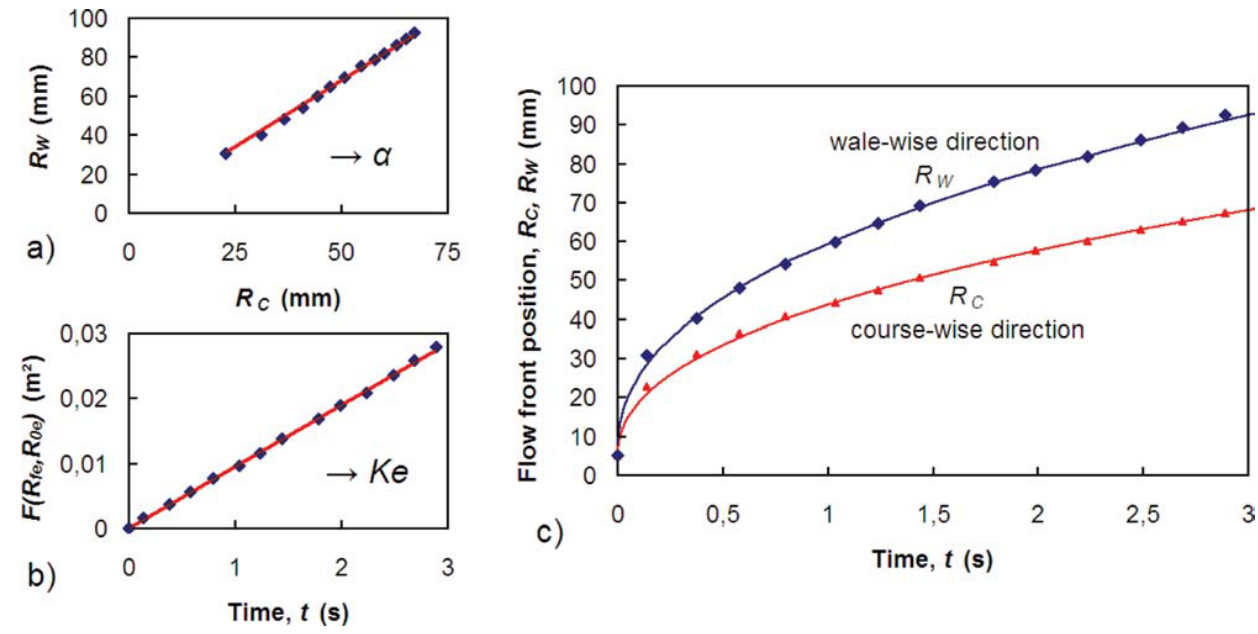

FIG. 5. The steps toward course- and wale-wise permeability identification: (a) anisotropy ratio determination, (b) equivalent isotropic permeability determination, (c) comparison of experimental and identified course- and wale-wise flow front position. [Color figure can be viewed in the online issue, which is available at wileyonlinelibrary.com.]

$$
\left\{\begin{array}{c}
\xi=\alpha^{\frac{1}{4}} \\
\eta=\alpha^{-\frac{1}{4}}
\end{array}\right.
$$

The value of the square root of the anisotropy ratio is determined by plotting $R_{\mathrm{fW}}$ against $R_{\mathrm{fC}}$ and calculating the least square slope of the experimental straight line (Fig. 5a), from which the values of $\xi, \eta$, and then $R_{\mathrm{fe}}$ are deduced. The equivalent permeability is determined by plotting the left term of $E q .2, F\left(R_{\mathrm{fe}}, R_{0 \mathrm{e}}\right)$, against time and calculating the slope of the obtained straight line (Fig. 5b) that depends on viscosity, pressure drop, fiber volume fraction, and equivalent permeability. The values of the wale- and course-wise permeability are then easily deduced from Eqs. 4 and 5.

Figure $5 \mathrm{c}$ compares the experimental flow front position in both directions and the values calculated using the equivalent isotropic system and the identified course- and wale-wise permeability. The analytical model fits very properly the experimental points, showing that the Darcy's law is valid in the present conditions.

\section{Measurement Error Analysis}

Equivalent permeability value $K_{\mathrm{e}}$ is estimated from $E q$. 2 , that can be rewritten:

$$
\begin{aligned}
& K_{\mathrm{e}}=\frac{\mu\left(1-V_{\mathrm{f}}\right)}{4 \delta P t} F\left(R_{\mathrm{fe}}, R_{0 \mathrm{e}}\right) \\
& F\left(R_{\mathrm{fe}}, R_{0 \mathrm{e}}\right)=R_{\mathrm{fe}}^{2}\left[2 \ln \left(\frac{R_{\mathrm{fe}}}{R_{0 \mathrm{e}}}\right)-1\right]+R_{0 \mathrm{e}}^{2}
\end{aligned}
$$

Logarithmic derivation of Eq. 6 allows the determination of the errors due to each term of the equation by a simple summation. If $\Delta X$ is the absolute error on the value of the parameter $X$, and $\Delta X / X$ is the relative error, then $E q .7$ is obtained.

$$
\begin{aligned}
& \frac{\Delta K_{\mathrm{e}}}{K_{\mathrm{e}}}=\frac{\Delta \mu}{\mu}+\frac{\Delta V_{\mathrm{f}}}{1-V_{\mathrm{f}}}+\frac{\Delta \delta P}{\delta P}+\frac{\Delta t}{t}+\frac{\Delta F\left(R_{\mathrm{fe}}, R_{0 \mathrm{e}}\right)}{F\left(R_{\mathrm{fe}}, R_{0 \mathrm{e}}\right)} \\
& \Delta F\left(R_{\mathrm{fe}}, R_{0 \mathrm{e}}\right)=4 R_{\mathrm{fe}} \ln \left(\frac{R_{\mathrm{fe}}}{R_{0 \mathrm{e}}}\right) \Delta R_{\mathrm{fe}}+2 R_{0 \mathrm{e}}\left(1-\frac{R_{\mathrm{fe}}^{2}}{R_{0 \mathrm{e}}^{2}}\right) \Delta R_{0 \mathrm{e}}
\end{aligned}
$$

Viscosity error is due to the error on the viscosity measurements $\left(\Delta \mu_{\mathrm{E}}= \pm 1 \mathrm{mPa} \cdot \mathrm{s}\right)$ fitted by the Arrhenius law $\left(E q .8\right.$ with $\mu_{0}=3.35 \times 10^{-7} \mathrm{~Pa} \cdot \mathrm{s}, E_{\mathrm{a}}=30.0 \times 10^{3}$ $\mathrm{J} \cdot \mathrm{mol}^{-1}$, and $T$ temperature in Kelvin, Fig. 3), and to the error on temperature measurement for each test $(\Delta T=$ $\pm 0.1 \mathrm{~K})$. The relative error on the viscosity is then close to $\pm 2.1 \%$.

$$
\begin{aligned}
& \mu=\mu_{0} e^{\frac{-E_{\mathrm{a}}}{R T}} \\
& \frac{\Delta \mu}{\mu}=\frac{\Delta \mu_{\mathrm{E}}}{\mu}+\frac{E_{\mathrm{a}}}{R T^{2}} \Delta T .
\end{aligned}
$$

Fiber volume fraction is estimated from sample mass and size and from fiber density (Eq.9), and relative error is the sum of the relatives errors of each material and sample property. The mass $m(98 \mathrm{~g})$ is measured with an accuracy $\Delta m=0.5 \mathrm{~g}$. The in-plane dimensions $L$ and $W$ are $200 \mathrm{~mm}$ measured with an accuracy $\Delta L=\Delta W=2$ $\mathrm{mm}$ (essentially due to shape error). Error on glass density $\rho_{\mathrm{g}}$ is neglected (the rounding error on the supplier data is lower than $0.4 \%$ ). The error on the thickness $e$ of the sample is the most important source of error. It is measured with an accuracy of $0.01 \mathrm{~mm}$ and a standard deviation lower than $0.04 \mathrm{~mm}$. These values lead to an absolute error $\Delta e= \pm 0.05 \mathrm{~mm}$ and a relative error of $\pm 1.4 \%$. The resulting relative error is then $\pm 3.9 \%$ on the fiber volume fraction and $\pm 1.4 \%$ on the porosity. 


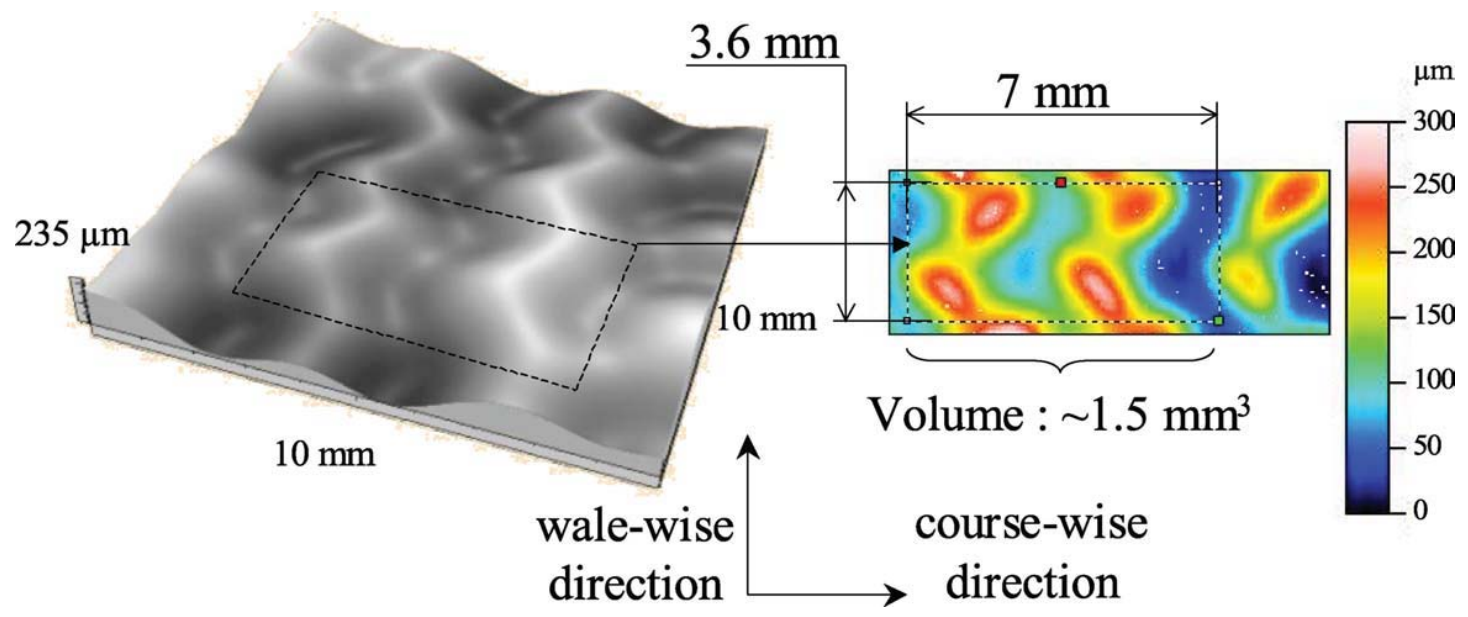

FIG. 6. Overestimation of the volume under the vacuum bag. [Color figure can be viewed in the online issue, which is available at wileyonlinelibrary. com.]

$$
\begin{aligned}
& V_{\mathrm{f}}=\frac{m}{\rho_{\mathrm{g}} L W e} \\
& \frac{\Delta V_{\mathrm{f}}}{V_{\mathrm{f}}}=\frac{\Delta m}{m}+\frac{\Delta \rho_{\mathrm{g}}}{\rho_{\mathrm{g}}}+\frac{\Delta L}{L}+\frac{\Delta W}{W}+\frac{\Delta e}{e}
\end{aligned}
$$

An other fiber volume fraction measurement error source is the knitted fabric surface macropores that are penetrated by the vacuum bagging (close to 100-150 $\mu \mathrm{m})$. This leads to an overestimation of the volume and an underestimation of the fiber volume fraction. To quantify this error, the surface weaviness of the vacuum bag was molded with model plaster and the replica was optically scanned using a chromatic confocal probe instrumented metrology platform (Fig. 6). The volume of the surface weaviness corresponding to this overestimation was evaluated to $1.5 \pm 0.1 \mathrm{~mm}^{3}$ on four elementary cells corresponding to an overestimation of the total volume of $1.95 \%$ and an underestimation of the fiber volume fraction of $1.91 \%$. Despite the low confidence in the repeatability of such a measurement, the error evaluated is large enough to be taken into account.

The pressure drop in the fibrous media is estimated by the difference between the atmospheric pressure $P_{\text {atm }}$ $(1013 \mathrm{hPa})$, measured with an accuracy $\Delta P_{\text {atm }}= \pm 100$ $\mathrm{Pa}$, and the vacuum pressure $P_{\mathrm{v}}(90 \mathrm{~Pa})$, measured with an accuracy $\Delta P_{\mathrm{v}}= \pm 5 \mathrm{~Pa}$. The error on this estimation is the sum of these two errors and of the hydrostatic pressure $\delta P_{\mathrm{h}}$ in the $\rho_{\text {fluid }}$ density fluid circuit and the pressure loss $\delta P_{\mathrm{p}}$ in the pipe, see $E q .10$ where $g$ is gravity acceleration. These two pressures cannot be compensated because they are not constant with time, but their order of magnitude could be estimated. The hydrostatic pressure in the circuit is minimized by setting the sample and the level of fluid in the oil tank at the same height. Nevertheless, the oil level $h$ in the tank varies of about $5 \mathrm{~mm}$ dur- ing the test and a variation $\Delta \delta P_{\mathrm{h}}= \pm 45 \mathrm{~Pa}$ can be estimated corresponding to a relative error of about $0.02 \%$. An analytical calculation of the unidirectional flow at the same flow rate in the pipe (Poiseuille flow) and in the fibrous medium (Darcy law) shows that in our experimental conditions, the error due to the pressure loss in the pipe is about $0.3 \%$ when the fluid has infiltrated $1 \mathrm{~mm}$ of the media, and lower than $0.015 \%$ when the fluid has infiltrated $20 \mathrm{~mm}$ of the media (the first flow front position measurement is generally close to $20 \mathrm{~mm}$ because of the injection inlet diameter). These results show that the error on the pressure drop in the sample due to the variation of the hydrostatic pressure and to the pressure loss in the pipe can be neglected. The relative error on the pressure drop is then close to $(0.1 \%)$.

$$
\begin{aligned}
& \delta P_{\mathrm{h}}=\rho_{\text {fluid }} \cdot g \cdot h \\
& \delta P=P_{\text {atm }}-P_{\mathrm{v}}-\delta P_{\mathrm{p}}+\delta P_{\mathrm{h}} \\
& \Delta \delta P=\Delta P_{\mathrm{atm}}+\Delta P_{\mathrm{v}}+\Delta \delta P_{\mathrm{p}}+\Delta \delta P_{\mathrm{h}}
\end{aligned}
$$

The accuracy on the time measurement $\Delta t$ is $\pm 0.05 \mathrm{~s}$ for a test duration of $5 \mathrm{~s}$ corresponding to a relative error of $\pm 1 \%$. The flow front position (the mean value is about $R=50 \mathrm{~mm})$ is measured with an accuracy $\Delta R_{\mathrm{f}}$ of \pm 0.5 $\mathrm{mm}$ and the error on the injection inlet radius $\left(R_{0}=5\right.$ $\mathrm{mm})$ is $\pm 0.1 \mathrm{~mm}$. As a consequence the relative error on $F$ function is calculated to be close to $1.5 \%$.

The repartition of the error sources in the permeability measurements are summarized in Table 1. The most important parameters are the fluid viscosity, and at the same level, the flow front position measurement and the fiber volume fraction evaluation. The improvement of the accuracy of these three parameters would require very important setup modifications for limited measurement accuracy amelioration. 
TABLE 1. Repartition of the error source in the permeability determination.

\begin{tabular}{|c|c|c|c|c|c|c|}
\hline Parameter $X$ & $\mu$ & $1-V_{\mathrm{f}}$ & $\delta P$ & $t$ & $F$ & $K_{\mathrm{e}}$ \\
\hline Relative error $\Delta X / X$ & $\pm 2.1 \%$ & $\pm 1.4 \%$ & $\pm 0.1 \%$ & $\pm 1 \%$ & $\pm 1.5 \%$ & $\pm 6.1 \%$ \\
\hline Mean value of $X$ & $0.06 \mathrm{~Pa} \cdot \mathrm{s}$ & $73 \%$ & $101,210 \mathrm{~Pa}$ & $5 \mathrm{~s}$ & $9,038 \mathrm{~mm}^{2}$ & $1.5 \times 10^{-9} \mathrm{~m}^{2}$ \\
\hline Absolute error $\Delta X$ & $\pm 1.2 \mathrm{mPa} \cdot \mathrm{s}$ & $\pm 1.0 \%$ & $\pm 105 \mathrm{~Pa}$ & $\pm 0.05 \mathrm{~s}$ & $\pm 131 \mathrm{~mm}^{2}$ & $\pm 9.2 \times 10^{-11} \mathrm{~m}^{2}$ \\
\hline
\end{tabular}

\section{Discussion on the Saturated Flow Assumption and Capillary Effects}

Previous analysis is valid assuming that fabric is saturated. One of the most important drawback of flow front monitoring methods is that the fibrous medium in the vicinity of the flow front is not saturated and that the volume of fluid in the fabric is then overestimated. Many authors have investigated the dual-scale behavior of the void formation in LCM processes [43]. The fibrous media are constituted by macropores between the yarns and micropores between the filaments into the yarns (Fig. 7). The infiltration phenomenon is then very sensitive to the infiltration speed, depending on the capillary velocity between the filaments. At low velocities, the capillary forces are predominant, and the flow takes place preferentially inside the yarn, whereas at high velocities, the flow takes place preferentially in the macropores, leading to the formation of microvoids inside the yarns. The existence of an optimal flow front velocity or, more generally, an optimal capillary number has been demonstrated experimentally $[44,45]$ and is used to minimize the micro- and macrovoids formation [46, 47]. Unfortunately, the present setup is not suitable to regulate flow-rate accurately because of the time response of the solenoid valve, whereas constant pressure experiments can be performed in conditions meeting identification method requirements. This section aims at verifying that the measurements have been performed in conditions where the volume of the voids could be neglected.

By modeling the yarn as a perfect hexagonal arrangement of filaments (Fig. 7), the fiber and micropore volumes can be estimated when the yarn length in the elementary cell is known. The volume of the macropores balances then the total volume. In the present case (cf. section 2.2 for the size of the yarns), the values estimated are $27 \%$ for the fiber volume fraction, $3 \%$ for the micropores volume fraction, and $70 \%$ for the macropores volume fraction. Even if the volume of the micropores may be underestimated with the assumption of perfect hexagonal arrangement of filaments, this calculation shows that the macropores are the most predominant in the measurement error of the fluid volume in the fabric due to unsaturated flow. The flow front velocity in the present experiment is between 10 and $50 \mathrm{~mm} \cdot \mathrm{s}^{-1}$, which is in the domain of low microvoids formation according to Labat et al. [44]. The corresponding modified capillary number used by Ruiz et al. [47] is between 0.02 and 0.1 in our case. With such a value, the microvoid formation accord- ing to Ruiz et al. [47] is then lower than 3\%. Even if the conditions of Ruiz work (infiltration of fiber glass mats by polyester resin) are not exactly the same as those of the present work (infiltration of fiber glass knit by canola oil), the properties of both fluids (viscosity of $0.06 \mathrm{~Pa} \cdot \mathrm{s}$, surface tension of $33.4 \pm 0.1 \mathrm{mN} \cdot \mathrm{m}^{-1}$, wetting angle of $35^{\circ}$ ) and both reinforcements (material and fiber fraction volume) are close enough to assess the complete infiltration of the macropores and neglect the volume of the micropores.

\section{Measurement Method Experimental Validation}

To validate the results of the measurements made with the method detailed in section 3.1 , the permeability of a $300 \mathrm{~g} \cdot \mathrm{m}^{-2}$ continuous glass filament mat has been measured and compared with Pomeroy et al. [48] results (steady state air permeability measurement), themselves match other bibliographical data. The present measurements have been performed on six layers mat with arbitrary orientation to improve the isotropy of the structure. The samples are $200 \times 200 \mathrm{~mm}^{2}$, and a 10-mm-diameter hole is cut under the injection inlet. The results are compared in Table 2 and show that, despite the difference of fluid viscosity, the present values are in the lower range of Pomeroy's values. The order of magnitude is the same as given in the literature $[49,50]$ for permeability measured with resin. This comparison validates the measurement setup and identification methodology, and even if permeability values are necessarily dispersed, they are reliable enough to study the influence on permeability of fabric deformation.

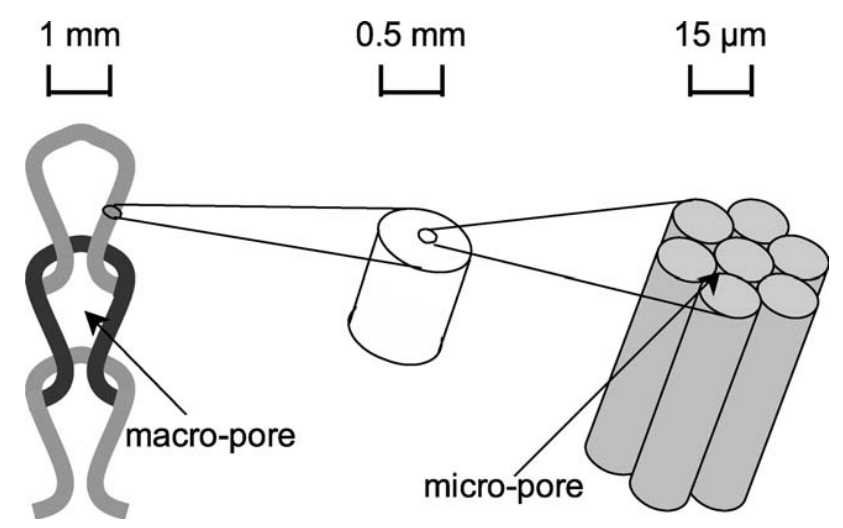

FIG. 7. Dual-scale porosity of knitted fabrics and perfect hexagonal arrangement assumption of yarn filaments. 
TABLE 2. Comparison of permeability measurements performed with the present setup and Pomeroy et al. [48] results.

\begin{tabular}{ccc}
\hline & \multicolumn{2}{c}{ Permeability $\left(10^{-10} \mathrm{~m}^{2}\right)$} \\
\cline { 2 - 3 } Fibre volume fraction $(\%)$ & Present study & Pomeroy et al. [48] \\
\hline 29.4 & $1.925 \pm 0.015$ & $2.75 \pm 0.55$ \\
30.4 & $1.615 \pm 0.025$ & $2.3 \pm 0.5$ \\
\hline
\end{tabular}

\section{EXPERIMENTAL RESULTS AND DISCUSSION}

Figure 8 shows the extension effect on permeability of the $1 \times 1$ rib knit fabric. The nonextended samples $(8 b)$ exhibit an anisotropic permeability with a ratio $\alpha=1.91$ between the wale-wise (higher permeability) and the course-wise (lower permeability) directions. The effect of the deformation is very significant on the anisotropy ratio, as can be observed on the elliptical flow front after an infusion time of $2 \mathrm{~s}$. If the wale-wise flow front position of both wale- and course-wise extended samples does not seem to be affected by the deformation, the course-wise flow front position significantly changes with the wale(8a) or course-wise (8c) extension, leading to an anisotropy ratio of 2.3 and 1.4 , respectively, for the wale- and course-wise extended samples.

Table 3 summarizes the results obtained for the three investigated preform configurations: nonextended, $15 \%$ course-wise extended and $7.5 \%$ wale-wise extended. Values of fiber volume fraction $V_{\mathrm{f}}$, in-plane deformations $\varepsilon_{\mathrm{W}}$ and $\varepsilon_{\mathrm{C}}$, transverse deformation $\varepsilon_{\tau}$ (evaluated from the thickness of the sample in the released and extended states), as well as equivalent $\left(K_{\mathrm{e}}\right)$, wale-wise $\left(K_{\mathrm{W}}\right)$, and course-wise $\left(K_{\mathrm{C}}\right)$ permeability, anisotropy ratio $\alpha$, and area density $d_{\mathrm{a}}$ are reported.

The equivalent permeability identification from six measurements performed on non-extended samples pro- vides a value of $13.6 \times 10^{-10} \mathrm{~m}^{2}$, and a value of permeability ratio of 1.91 for a fiber volume fraction of $27.6 \%$. The mean permeability of nonextended samples is 9.84 $\times 10^{-10} \mathrm{~m}^{2}$ in the course-wise direction and $18.8 \times$ $10^{-10} \mathrm{~m}^{2}$ in the wale-wise direction, with a standard deviation about $8.5 \%$. Figure 9 reports the experimental permeability values and anisotropy ratio for the three experimental configurations: when compared with nonextended samples, course-wise extension increases permeability values and decreases anisotropy ratio, whereas wale-wise extension decreases permeabilities and increases anisotropy.

\section{Fiber Volume Fraction and Equivalent Permeability}

It can be noticed in Table 3 that fiber volume fraction does not change in the same way when extending in wale- $(1.8 \%$ increase) or course-wise $(4.7 \%$ decrease $)$ direction. Considering that fiber volume fraction and permeability may be strongly connected, a first dependence mechanism to the deformation can be established.

Figure 10a summarizes mean equivalent, wale- and course-wise permeability values with respect to fiber volume fraction, to quantify the effect of deformation on fiber volume fraction and fabric permeability. It is interesting to underline that the error areas on such graphs are not rectangles, but parallelograms due to the interdependence of permeability and fiber volume fraction errors. The experimental data exhibit a non-negligible dispersion that can be reduced by analyzing the results through the Kozeny and Carman equation (Eq. 11 [51]) to compensate the effect of fiber volume fraction variation. This equation is deduced from the resolution of the Navier-Stokes equations in channels of different cross sections. In this equation, $r_{\mathrm{f}}$ is the radius of the filaments $(6.5 \mu \mathrm{m}$ in our case $)$ and $c$ is the so-called Kozeny constant, characterizing the

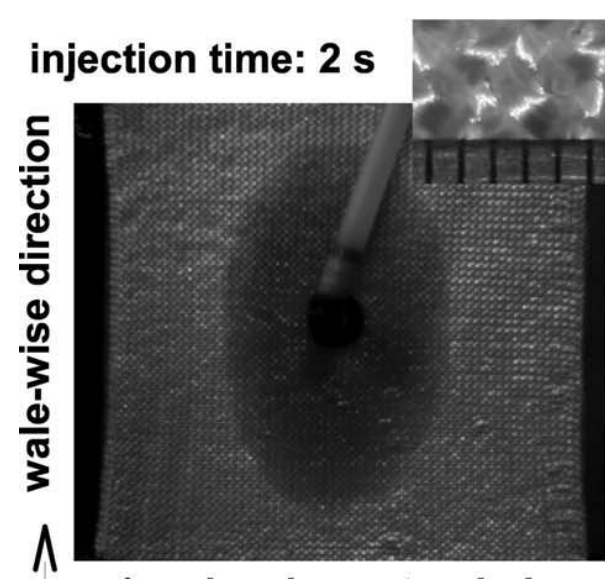

a) wale-wise extended course-wise direction

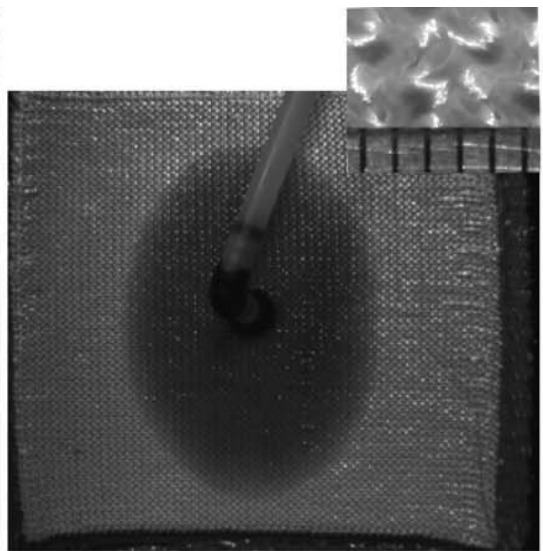

b) non-extended

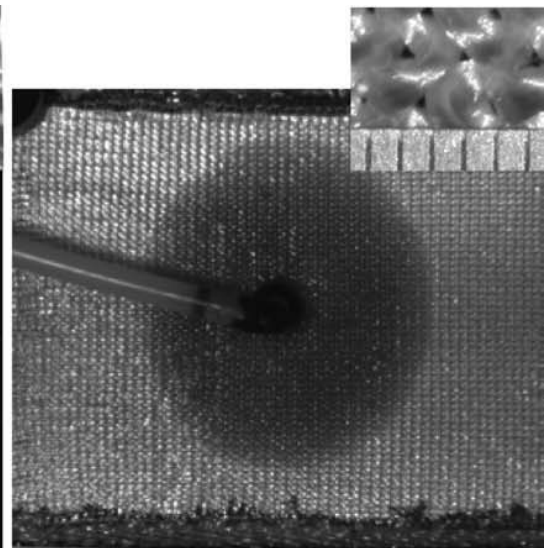

c) course-wise extended $100 \mathrm{~mm}$

FIG. 8. Effect of wale- (a) and course-wise (c) extension on $1 \times 1$ rib knit fabric permeability (insets show elementary cells: 1 division for 1 mm). 
TABLE 3. Sample configuration and test results.

\begin{tabular}{|c|c|c|c|c|c|c|c|c|c|c|}
\hline Sample & & $V_{\mathrm{f}}(\%)$ & $\varepsilon_{\mathrm{W}}(\%)$ & $\varepsilon_{\mathrm{C}}(\%)$ & $\varepsilon_{\tau}(\%)$ & $K_{\mathrm{e}}\left(10^{-10} \mathrm{~m}^{2}\right)$ & $\alpha$ & $K_{\mathrm{C}}\left(10^{-10} \mathrm{~m}^{2}\right)$ & $K_{\mathrm{W}}\left(10^{-10} \mathrm{~m}^{2}\right)$ & $d_{\mathrm{a}}\left(\mathrm{g} \cdot \mathrm{m}^{-2}\right)$ \\
\hline \multirow[t]{8}{*}{ Nonextended } & 1 & 27.6 & 0.00 & 0.00 & -19.6 & 13.8 & 2.05 & 9.63 & 19.8 & 2,405 \\
\hline & 2 & 27.7 & 0.00 & 0.00 & -20.0 & 12.9 & 1.98 & 9.20 & 18.2 & 2,405 \\
\hline & 3 & 26.7 & 0.00 & 0.00 & -18.6 & 15.3 & 1.93 & 11.0 & 21.2 & 2,393 \\
\hline & 4 & 28.2 & 0.00 & 0.00 & -20.9 & 12.1 & 1.81 & 8.97 & 16.3 & 2,418 \\
\hline & 5 & 27.9 & 0.00 & 0.00 & -19.1 & 14.4 & 1.85 & 10.6 & 19.6 & 2,471 \\
\hline & 6 & 27.8 & 0.00 & 0.00 & -19.4 & 13.0 & 1.83 & 9.62 & 17.6 & 2,441 \\
\hline & Mean & 27.6 & 0.00 & 0.00 & -19.6 & 13.6 & 1.91 & 9.84 & 18.8 & 2,422 \\
\hline & SD & 0.490 & 0.00 & 0.00 & 0.792 & 1.15 & 0.0947 & 0.798 & 1.76 & 29.0 \\
\hline \multirow{6}{*}{ Course-wise extended } & 7 & 26.4 & -7.69 & 14.3 & -21.0 & 18.8 & 1.48 & 14.4 & 22.9 & 2,280 \\
\hline & 8 & 26.4 & -12.8 & 16.7 & -18.5 & 18.5 & 1.38 & 15.8 & 21.7 & 2,389 \\
\hline & 9 & 26.6 & -10.3 & 14.3 & -19.1 & 19.3 & 1.42 & 16.2 & 23.0 & 2,345 \\
\hline & 10 & 25.9 & -7.89 & 15.2 & -17.6 & 18.3 & 1.34 & 15.8 & 21.3 & 2,330 \\
\hline & Mean & 26.3 & -9.67 & 15.1 & -19.1 & 18.7 & 1.41 & 15.6 & 22.2 & 2,336 \\
\hline & $\mathrm{SD}$ & 0.299 & 2.40 & 1.13 & 1.44 & 0.435 & 0.0597 & 0.790 & 0.854 & 45.0 \\
\hline \multirow[t]{7}{*}{ Wale-wise extended } & 11 & 27.1 & 7.69 & -4.76 & -19.0 & 11.6 & 2.38 & 7.51 & 17.9 & 2,345 \\
\hline & 12 & 27.8 & 8.80 & -4.76 & -22.2 & 12.5 & 2.37 & 8.14 & 19.3 & 2,345 \\
\hline & 13 & 28.7 & 7.33 & -4.76 & -22.2 & 9.86 & 2.12 & 6.77 & 14.4 & 2,402 \\
\hline & 14 & 28.3 & 6.77 & -2.38 & -23.3 & 8.93 & 2.54 & 5.59 & 14.3 & 2,344 \\
\hline & 15 & 28.7 & 6.70 & -7.14 & -20.9 & 12.3 & 2.16 & 8.39 & 18.1 & 2,440 \\
\hline & Mean & 28.1 & 7.46 & -4.76 & -21.5 & 11.0 & 2.31 & 7.28 & 16.8 & 2,375 \\
\hline & $\mathrm{SD}$ & 0.680 & 0.854 & 1.68 & 1.65 & 1.57 & 0.173 & 1.13 & 2.30 & 43.9 \\
\hline
\end{tabular}

arrangement of the fibers of a given fabric in a given direction.

$$
K=\frac{r_{\mathrm{f}}^{2}}{4 c} \frac{\left(1-V_{\mathrm{f}}\right)^{3}}{V_{\mathrm{f}}^{2}}
$$

Wale-wise permeability varies with the deformation, but the Kozeny-Carman curve (see Fig. 10a) shows that this variation is mainly due to the variation of the fiber volume fraction, whereas it is not sufficient to explain the variations of the course-wise permeability. As a matter of fact, if all the wale-wise permeability values can be fitted by the same Kozeny-Carman curve $(c=0.028)$, the course-wise permeability fitting requests at least three curves (i.e. three $c$ values) depending on the deformation level. It is noteworthy that the equivalent permeability is

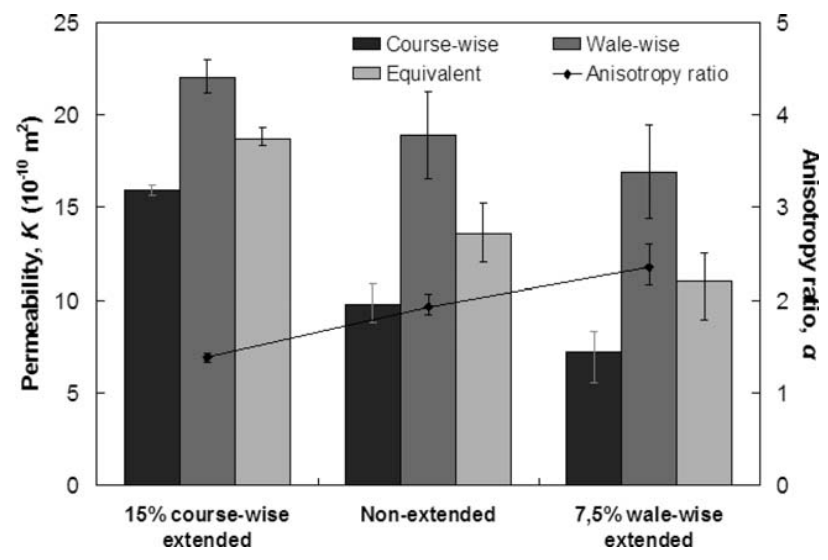

FIG. 9. Course-wise, wale-wise, equivalent permeability, and anisotropy ratio results (error bars are minimal and maximal measured values).

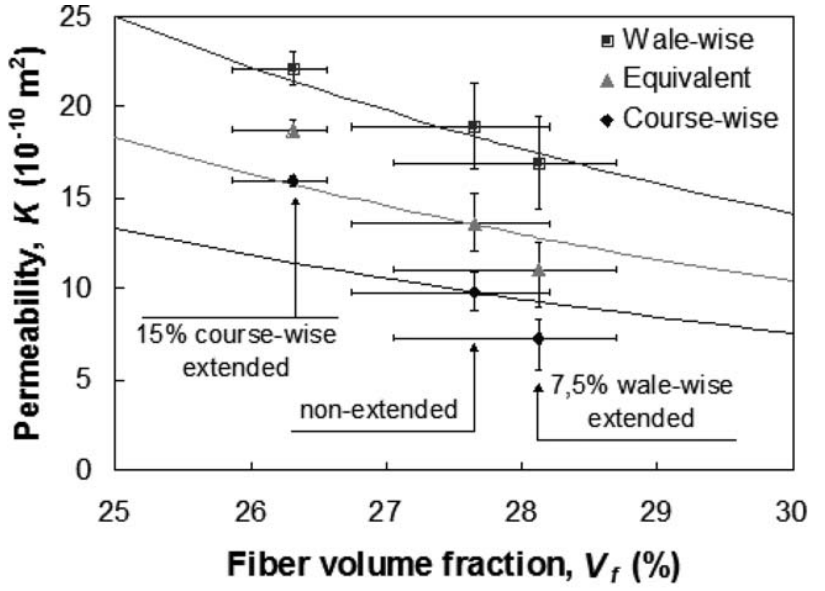

a)

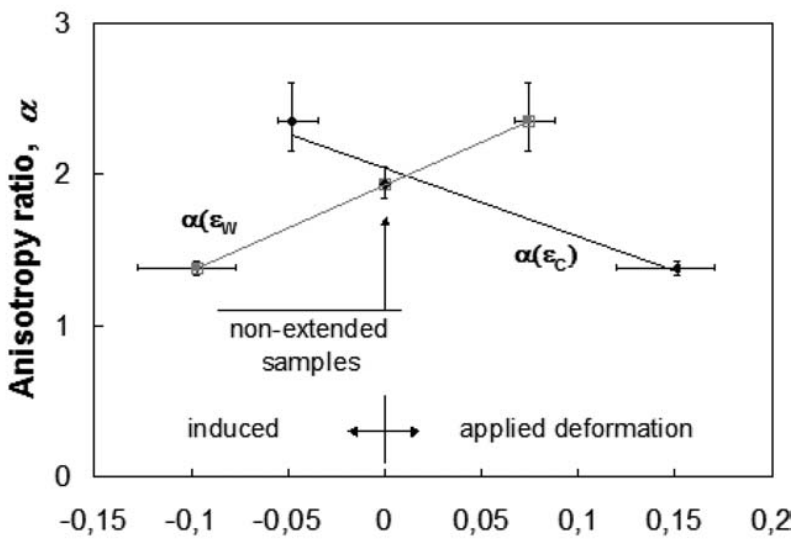

b) Course- $\left(\varepsilon_{c}\right)$ or wale- $\left(\varepsilon_{w}\right)$ wise deformation

FIG. 10. Effect of deformation on fiber volume fraction, equivalent, wale- and course-wise permeability (a) and anisotropy ratio (b) (error bars are minimal and maximal measured values). 

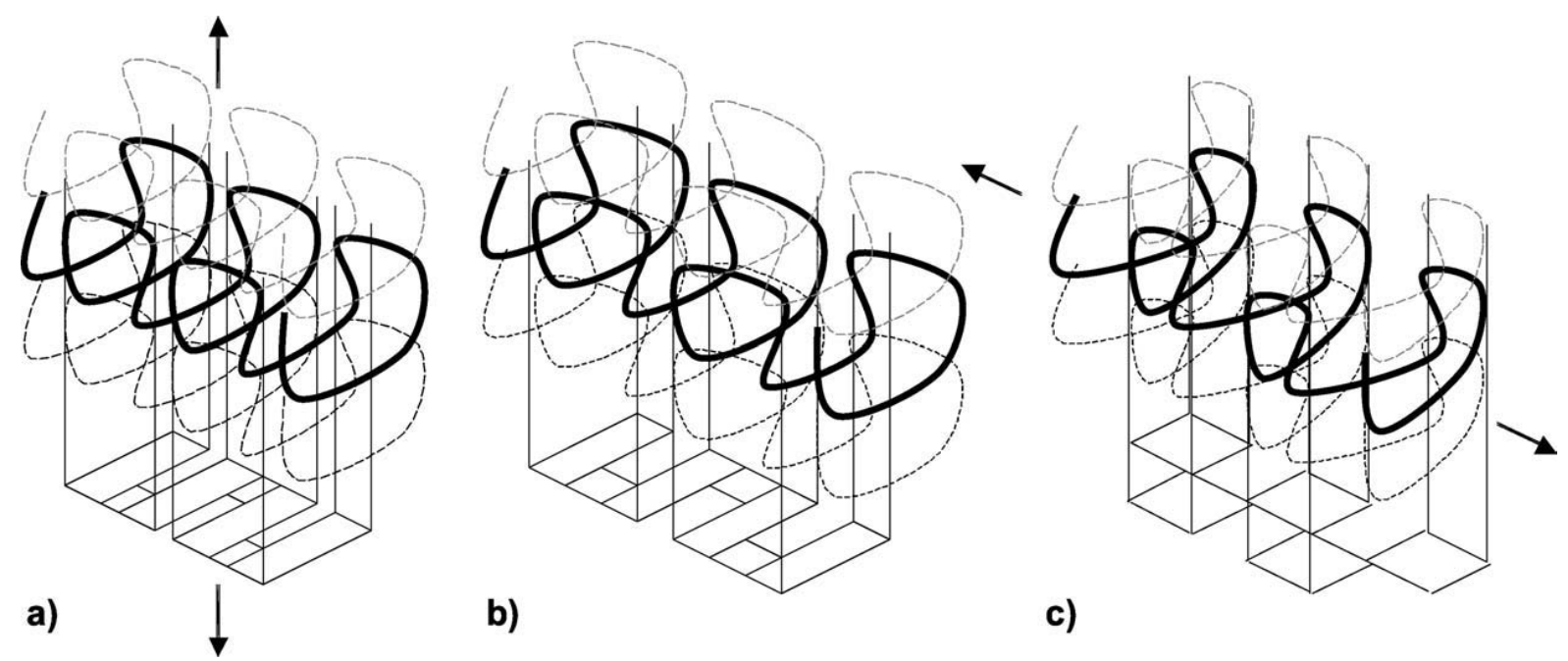

c)

FIG. 11. Schematized view of deformation effect on the knit architecture cross section: (a) wale-wise extension, (b) no extension, and (c) course-wise extension.

dependent to the deformation (cf. Fig. 10a), as the experimental results could not be properly fitted by a single Kozeny-Carman curve. Figure 10b shows that a linear fit describes properly the evolution of $\alpha$ with the wale-wise deformation in the investigated cases, whereas it describes less properly the evolution of $\alpha$ with the course-wise deformation. An appropriate way to interpolate the $1 \times 1$ rib knit fabric permeability while in-plane extension seems to be a Kozeny Carman fit of the wale-wise permeability (Eq. 11 with $c=0.028)$ and a linear fit of the anisotropy ratio as a function of the wale-wise deformation.

\section{Anisotropy of the $1 \times 1$ Rib Knit Permeability and Mesoscale Structure Geometry}

The experimental results detailed earlier underline the anisotropy of the $1 \times 1$ rib knit structure permeability and its evolution with the fabric deformation. By analyzing the knitted structure at the mesoscopic scale (yarn and elementary cell scale), it can be observed that the structure is not as flat as shown in Fig. 1, but exhibits a complex 3D geometry (Fig. 11b): the yarn segment joining two consecutive front and back loops is not course-wise but through-thickness oriented. This leads to the occurrence of wale-wise oriented rich fiber zones that are separated by poor fiber zones constituted of course-wise oriented yarn segments.

This fabric structure explains why fluid flows preferentially in the poor fiber wale-wise oriented channel direction leading to a higher permeability value, whereas fluid is slowed in the course-wise direction when crossing the rich fiber areas.

Fabric deformation in the wale-wise direction does not change significantly the configuration of the elementary cell (Figs. 8a and 11a) and impact on permeability is then very weak and essentially due to the modification of the fiber volume fraction and the size of the poor and rich fiber areas described previously. On the contrary, the course-wise de- formation modifies the structure more significantly (Fig. 8c and 11c): the extension tightens the front and back loops aligned on one or other side of the sample. A microchannel without any fibers appears then on each side. This channel is wale-wise oriented and does not modify significantly the wale-wise mean permeability: the poor fiber areas whose density have been increased by the deformation are replaced by these channels. On the contrary, the fluid can flow in the course-wise direction from a channel toward the next without crossing any rich fiber area. Thus, the permeability is widely modified by the deformation due to the creation of these channels and the alignment of most of the fibers in the course-wise direction.

\section{CONCLUSION}

Mechanical properties of knitted fabric reinforced composite materials can be improved by preform extension before liquid injection. Nevertheless, this induces a modification of fabric permeability and processing conditions. In this work, $1 \times 1$ rib knit samples have been extended in the course- and wale-wise direction and their in-plane principal permeability values have been measured simultaneously using a 2D flow front monitoring method. An error analysis is detailed that shows that the experimental results dispersion is difficult to improve because of the intrinsic variability of fibrous materials and composite processing. Nevertheless, it is low enough to highlight the effect of deformation.

Deformation and fiber volume fraction effects on permeability are discriminated by Kozeny-Carman results fitting. Results show that the effect of the sample deformation is very significant on the course-wise permeability, whereas the wale-wise permeability is less sensitive. Wale-wise permeability evolution is mainly related to fiber volume fraction changes. Deformation has a little influence on the equivalent isotropic permeability of the 
extended and nonextended samples, but highly modifies the anisotropy ratio. Finally, the elementary cell mesostructure is described to give a qualitative explanation of fabric anisotropic permeability and the deformation induced permeability changes.

\section{ACKNOWLEDGMENTS}

The authors greatly acknowledge Textile Aéro Tarn for the knitted samples delivery and especially Mr. Bernard Cabanes, Nicolas Dumont, and Francis Planel.

\section{REFERENCES}

1. A.W. Van Vuure, F.K. Ko, and C. Beevers, Textile Res. J., 73, 1 (2003).

2. S. Savci, J.I. Curiskis, and M.T. Pailthorpe, Compos. Sci. Tech., 60, 1931 (2000).

3. L. Balea, G. Dusserre, G. Bernhart, and N. Dumont, Proc. JNC 16, Toulouse, France, hal-00391023 (2009).

4. K. H. Leong, M. Nguyen, and I. Herszberg, J. Mater. Sci., 34, 2377 (1999).

5. O.A. Khondker, K.H. Leong, and I. Herszberg, Compos. Part A, 32, 1513 (2001).

6. S.V. Lomov, G. Huysmans, Y. Luo, R.S. Parnas, A. Prodromou, I. Verpoest, and F.R. Phelan, Compos. Part A, 32, 1379 (2001).

7. S.V. Lomov, M. Barburski, T. Stoilova, I. Verpoest, R. Akkerman, R. Loendersloot, and R.H. W. ten Thije, Compos. Part A, 36, 1188 (2005).

8. K. Buet-Gautier and P. Boisse, Exp. Mech., 41, 260 (2001).

9. Y. Luo and I. Verpoest, Compos. Part A, 33, 197 (2002).

10. M. de Araùjo, R. Fangueiro, and H. Hong, AUTEX Res. J., 3, 166 (2003).

11. S. Comas-Cardona, C. Binetruy, and P. Krawczak, Compos. Sci. Tech., 67, 638 (2007).

12. P. Boisse, B. Zouari, and J.L. Daniel, Compos. Part A, 37, 2201 (2006).

13. B. Zhu, T.X. Yu, and X.M. Tao, Compos. Sci. Tech., 67, 252 (2007).

14. B. Verleye, R. Croce, M. Griebel, M. Klitz, S.V. Lomov, G. Morren, H. Sol, I. Verpoest, and D. Roose, Compos. Sci. Tech., 68, 2804 (2008).

15. F. Loix, P. Badel, L. Orgéas, C. Geindreau, and P. Boisse, Compos. Sci. Tech., 68, 1624 (2008).

16. C. Demaria, E. Ruiz, and F. Trochu, Polym. Compos., 28, 797 (2007).

17. A. Nabovati, E.W. Llewellin, and A.C.M. Sousa, Compos. Part A, 40, 860 (2009).

18. B. Verleye, S.V. Lomov, A. Long, I. Verpoest, and D. Roose, Compos. Part A, 41, 29 (2010).

19. C.C. Wong, A.C. Long, M. Sherburn, F. Robitaille, P. Harrison, and C.D. Rudd, Compos. Part A, 37, 847 (2006).

20. C. Lekakou, S. Edwards, G. Bell, and S.C. Amico, Compos. Part A, 37, 820 (2006).

21. G. Morren, M. Bottiglieri, S. Bossuyt, H. Sol, D. Lecompte, B Verleye, and S.V. Lomov, Compos. Part A, 40, 244 (2009).
22. C. Demaria, E. Ruiz, and F. Trochu, Polym. Compos., 28, 812 (2007).

23. A. Endruweit and P. Ermanni, Compos. Part A, 35, 439 (2004).

24. F. Loix, L. Orgéas, C. Geindreau, P. Badel, P. Boisse, and J.F. Bloch, Compos. Sci. Tech., 69, 612 (2009).

25. H. Darcy, Les fontaines publiques de la ville de Dijon, V. Dalmont Paris (1856).

26. N. Kuentzer, P. Simacek, S.G. Advani, and S. Walsh, Compos. Part A, 37, 2057 (2006).

27. J.M. Lawrence, V. Neacsu, and S.G. Advani, Compos. Part A, 40, 1053 (2009).

28. J.M. Bayldon and I.M. Daniel, Compos. Part A, 40, 1044 (2009).

29. J.M. Lawrence, J. Barr, R. Karmakar, and S.G. Advani, Compos. Part A, 35, 1393 (2004).

30. K.K. Han, C.W. Lee, and B.M. Rice, Compos. Sci. Tech., 60, 2435 (2000).

31. G. Morren, S. Bossuyt, and H. Sol, Compos. Part A, 39, 1530 (2008).

32. Q. Liu, R.S. Parnas, and H.S. Giffard, Compos. Part A, 38, 954 (2007).

33. P.B. Nedanov and S.G. Advani, J. Compos. Mater., 36, 241 (2002).

34. D.Z. Turner and K.D. Hjelmstad, Compos. Part B, 36, 609 (2005).

35. R. Gantois, E. Jourdain, and G. Dusserre, Recent Pat. Eng., 3, 109 (2009).

36. W.J. Shanahan and R. Postle, Textile Res. J., 43, 532 (1973).

37. M. Hautier, D. Lévêque, C. Huchette, and P. Olivier, Proc. JNC 16, Toulouse, France, hal-00389232 (2009).

38. Y. Ma and R. Shishoo, J. Compos. Mater., 33, 729 (1999).

39. Y. Luo, I. Verpoest, K. Hoes, M. Vanheule, H. Sol, and A. Cardon, Compos. Part A, 32, 1497 (2001).

40. D.A. Steenkamer, S.H. McKnight, D.J. Wilkins, and V.M. Karbhari, J. Mater. Sci., 30, 3207 (1995).

41. J.R. Weitzenböck, R.A. Shenoi, and P.A. Wilson, Compos. Part A, 30, 781 (1999).

42. K.L. Adams, W.B. Russel, and L. Rebenfeld, Int. J. Multiphase Flow, 14, 203 (1988).

43. J. Bréard, Y. Henzel, F. Trochu, and R. Gauvin, Polym. Compos., 24, 391 (2003).

44. L. Labat, J. Bréard, S. Pillut-Lesavre, and G. Bouquet, Eur. Phys. J., Appl. Phys., 16, 157 (2001).

45. B. Gourichon, C. Binetruy, and P. Krawczak, Compos. Sci. Tech., 66, 976 (2006).

46. F. Trochu, E. Ruiz, V. Achim, and S. Soukane, Compos. Part A, 37, 890 (2006).

47. E. Ruiz, V. Achim, S. Soukane, F. Trochu, and J. Bréard, Compos. Sci. Tech., 66, 475 (2006).

48. R. Pomeroy, S. Grove, J. Summerscales, Y. Wang, and A. Harper, Compos. Part A, 38, 1439 (2007).

49. H. Golestanian, J. Mater. Sci., 43, 6676 (2008).

50. Y.J. Lee, J.H. Wu, Y. Hsu, and C.H. Chung, Polym. Compos., 27, 665 (2006).

51. P.C. Carman, Trans. Inst. Chem. Eng., 15a, 150 (1937). 